\title{
Descemet's stripping automated endothelial keratoplasty in patient with iridocorneal endothelial syndrome.
}

\author{
Abdullah Albahlal* \\ Department of Ophthalmology, King Khaled Eye Specialist Hospital, Riyadh, Saudi Arabia
}

\begin{abstract}
One eye with ICE syndrome underwent DSAEK for corneal decompensation after cataract surgery and was followed up for $\mathbf{2 4}$ months. DSAEK was successfully performed in this case. No graft dislocation occurred. At first post-operative visit patient showed visual improvement to 20/160 with pinhole. IOP: 10, corneal clarity has significantly improved. At 6 months, VAPH was 20/200 and corneal clarity allowed to treat significant posterior capsular opacity then VAPH improved to 20/40. At last follow up, with correction of the compound hyperopic astigmatism the patient reached VA 20/60, normal IOP, and stable iris appearance. In conclusion, Simple DSEK might be a safe option for ICE patients with pseudophakic corneal decompensating.
\end{abstract}

Keywords: Opthalmology, dsaek, keratoplasy, ice syndrome, iridocorneal endothelial syndrome.

Accepted on September 12, 2018

\section{Introduction}

Iridocorneal Endothelial (ICE) syndrome is a rare disorder that is characterized by structural and proliferative abnormalities of the corneal endothelium, iris anomalies, and obstruction of the iridocorneal angle [1]. These changes cause many consequent pathologies as decompensated cornea and glaucoma [2]. Ice syndrome includes many different conditions: Cogan-Reese syndrome, progressive essential iris atrophy, and Chandler syndrome [3]. ICE syndrome IS mostly diagnosed in adults, females are more commonly encountered than males [4]. The management ICE syndrome has been debatable for a long time. Results of Penetrating Keratoplasty (PK) had high rates of endothelial failure [5]. Descemet stripping automated endothelial keratoplasty has had great results in managing endothelial dysfunction over the past decade. Large case series mainly involving patients with Fuchs Endothelial Dystrophy (FED) have proven that DSEK and DSAEK are less-invasive than PK with better 1 visual results and faster recovery with better endothelial survival [6-8].

\section{Case Report}

A 65 year-old woman medically free presented with one eye with ICE syndrome (Figure 1). She underwent DSAEK for corneal decompensation after cataract surgery and was followed up for 24 months. The pupil was constricted preoperatively. The anterior chamber maintainer was used intraoperatively. DSAEK lenticule was prepared and then injected utilizing Busin Glide. Air bubble was introduced into anterior chamber to keep the lenticule attached. Best corrected visual acuity, Intraocular pressure, corneal clarity and astigmatism were recorded preoperatively and at 6,12 , and 24 months postoperatively. DSAEK was successfully performed in this case. No graft dislocation occurred. Preoperative VA was HM, IOP was 20 $\mathrm{mmHg}$, and cornea was too edematous preventing assessment of other AS structures. B-scan Ultrasonography showed normal posterior segment. At first post-operative visit patient showed visual improvement to 20/160 with pinhole (Figure 2). IOP: 10, corneal clarity has significantly improved. At 6 months, VAPH was $20 / 200$ and corneal clarity allowed to treat significant posterior capsular opacity then VAPH improved to 20/40. It also allowed diagnosis of ICE syndrome with the characteristic iris hypoplasia, corectopia and peripeheral anterior synechia. At last follow up, with correction of the compound hyperopic astigmatism the patient reached VA 20/60, normal IOP, and stable iris appearance.

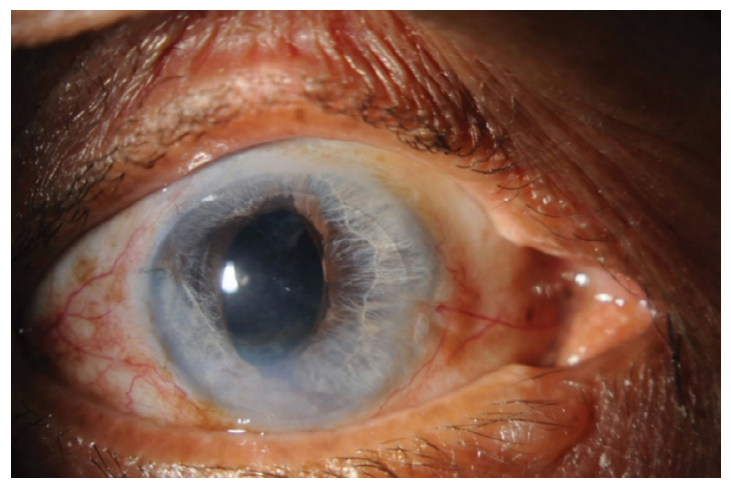

Figure 1. Eye with ICE syndrome.

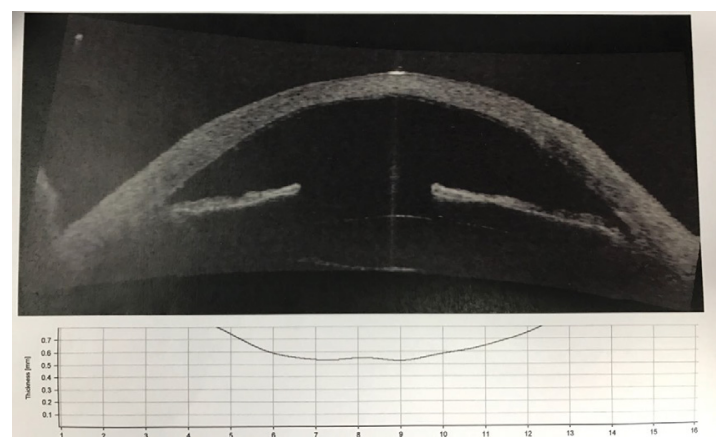

Figure 2. B-scan ultrasonography.

\section{Discussion}

DSAEK was successfully performed in this case. No graft dislocation occurred. Preoperative VA was HM, IOP was 20 $\mathrm{mmHg}$, cornea was too edematous preventing assessment of 
other AS structures. B-scan Ultrasonography showed normal posterior segment. At first post-operative visit patient showed visual improvement to 20/160 with pinhole. IOP: 10 , corneal clarity has significantly improved. At 6 months, VAPH was $20 / 200$ and corneal clarity allowed to treat significant posterior capsular opacity then VAPH improved to 20/40. Endothelial graft survival in ICE syndrome is still not fully known, however, the earlier outcomes are better. Studies of follow up after 24 months are not available [9-12]. Despite all the challenges associated with eyes having ICE syndrome, the short-term results were favorable. There were no primary graft failures and no graft detachments or dislocations. One of the case series showed that The long-term results were disappointing. The most limiting factor for graft survival was perioperative endothelial cell loss, with a mean 3-month cell loss of 55\% and 6-month cell loss of $78 \%$ [13]. Despite a relatively good prognosis and outcomes associated with ICE syndrome, glaucoma control is crucial for long-term success [14]. If there is a high probability the graft need to be replaced in patients with the tube in the anterior chamber. An important advantage of DSAEK compared with PK in such cases is that, should a regraft become necessary, an original DSAEK graft can just be stripped out by pulling on one edge and a new donor button can be placed without damage to the overlying host corneal structure or the need for corneal sutures, the PK is on the other hand more traumatic to the cornea. DSAEK could be used after using PK to restore the clarity [15].

\section{Conclusion}

DSAEK obviously has many different pros of better maintenance of the structure of the cornea and better visual outcome compared with PK, all of these advantages are there among ICE syndrome eyes. Regrafting after DSAEK is easier than after PK. Earlier Rejection may also be less common with DSAEK, it is essential to keep in mind for patients going for endothelial keratoplasty, the long-term outcomes, and long follow up and risk of repeated operations. But generally, Simple DSAEK might be a safe option for ICE patients with psudophakic corneal decompensation.

\section{Conflict of Interest}

The authors declare there is no conflict of interest.

\section{References}

1. Shields MB. Progressive essential iris atrophy, Chandler's syndrome, and the iris nevus (Cogan-Reese) syndrome: A spectrum of disease. Surv Ophthalmol. 1979;24:3-20.

2. Laganowski HC, Muir MGK, Hitchings RA. Glaucoma and the iridocorneal endothelial syndrome. Arch Ophthalmol. 1992;110:346-50.

3. Eagle RC, Font RL, Yanoff $M$, et al. Proliferative endotheliopathy with iris abnormalities. The iridocorneal endothelial syndrome. Arch Ophthalmol. 1979;97:2104-11.

4. Salim S, Shields MB, Walton D. Iridocorneal endothelial syndrome in a child. J Pediatr Ophthalmol Strabismus. 2006;43:308-10.

5. De Broff BM, Thoft RA. Surgical results of penetrating keratoplasty in essential iris atrophy. J Refract Corneal Surg. 1994;10:428-32.

6. Terry MA, Shamie N, Chen ES, et al. Endothelial keratoplasty: The influence of preoperative donor endothelial cell densities on dislocation, primary graft failure, and 1-year cell counts. Cornea. 2008;27:1131-37.

7. Price FW Jr, Price MO. Descemet's stripping with endothelial keratoplasty in 200 eyes: Early challenges and techniques to enhance donor adherence. J Cataract Refract Surg. 2006;32:411-8.

8. Price MO, Price FW Jr. Endothelial cell loss after descemet stripping with endothelial keratoplasty influencing factors and 2-year trend. Ophthalmology. 2008;115:857-65.

9. Price MO, Price FW Jr. Descemet stripping with endothelial keratoplasty for treatment of iridocorneal endothelial syndrome. Cornea. 2007;26:493-7.

10. Chaurasia S, Ramappa M, Garg P, et al. Endothelial keratoplasty in the management of irido-corneal endothelial syndrome. Eye (Lond). 2013;27:564-6.

11. Kymionis GD, Kontadakis GA, Agorogiannis GI, et al. Descemet stripping automated endothelial keratoplasty combined with phacoemulsification in Chandler syndrome. Eur J Ophthalmol. 2011;21:495-7.

12. Bahar I, Kaiserman I, Buys Y, et al. Descemet's stripping with endothelial keratoplasty in iridocorneal endothelial syndrome. Ophthalmic Surg Lasers Imaging. 2008;39:54-6.

13. Price MO, Gorovoy M, Price FW Jr, et al. Descemet's stripping automated endothelial keratoplasty: three-year graft and endothelial cell survival compared with penetrating keratoplasty. Ophthalmology. 2013;120:246-51.

14. Price FW Jr, Wellemeyer M. Long-term results of Molteno implants. Ophthalmic Surg. 1995;26:130-35.

15. Price FW Jr, Price MO. Endothelial keratoplasty to restore clarity to a failed penetrating graft. Cornea. 2006;25:895-99.

\section{*Correspondence to:}

Abdullah Albahlal

Department of Ophthalmology

King Khaled Eye Specialist Hospital

Riyadh

Saudi Arabia

Tel: 0555414113

E-mail: aalbahla191@gmail.com 